\title{
Gestión de llantas usadas en municipios de baja complejidad de la Provincia Centro del departamento de Boyacá, Colombia
}

\author{
Management of Used Tires in Low Complexity Municipalities in \\ the Central Province of the Department of Boyacá, Colombia
}

\section{Andrea Angélica Bernal-Figueroa ${ }^{\mathrm{ac}}$, Zulma Edelmira Rocha-Gilad, Leidy Paola Buitrago-Ramírez ${ }^{\mathrm{b}}$}

\footnotetext{
a Grupo de Investigación Gestión Ambiental, Programa de Ingeniería Ambiental, Facultad de Ciencias e Ingeniería, Universidad de Boyacá, Colombia.

${ }^{\mathrm{b}}$ Ingeniera ambiental | https://orcid.org/0000-0002-5456-259X

c aabernal@uniboyaca.edu.co|https://orcid.org/0000-0001-9024-5666

${ }^{d}$ https://orcid.org/0000-0001-8331-4707
}

\section{RESUMEN}

En Colombia las llantas usadas no son consideradas un residuo peligroso. Sin embargo, estas se componen de sustancias con dicha connotación, las cuales generan impactos negativos sobre la salud y el ambiente si no se realiza un manejo adecuado. Aunque en Colombia se han generado instrumentos normativos de sistemas de recolección selectiva, se tiene escaso conocimiento de aspectos relacionados con su gestión, generación y manejo; situación que se acrecienta en municipios de baja complejidad, donde a pesar de su generación no se realiza seguimien-

Citation: Bernal-Figueroa, A. A., Rocha-Gil, Z. E. y Buitrago-Ramírez, L. P. (2021). Gestión de llantas usadas en municipios de baja complejidad de la Provincia Centro del departamento de Boyacá, Colombia. Mutis, 11(2), 96108. https://doi.org/10.21789/22561498.1767

Recibido: 8 de junio de 2021. Aceptado: 19 de julio de 2021.

Copyright: $\odot 2021$. Bernal-Figueroa, A. A., Rocha-Gil, Z. E. y BuitragoRamírez, L. P. (2021). This is an open-access article, which permits unrestricted use, distributions and reproduction in any medium, provided the original author and source are credited.

Competing Interests: The authors have no conflict of interest. to y la oferta de empresas de recolección o centros de acopio es limitada. Por ello, se realizó un estudio sobre la gestión de llantas usadas en municipios de baja complejidad de la Provincia Centro del departamento de Boyacá, con el fin de indagar acerca de su generación, disposición y manejo, al igual que sobre el conocimiento de programas de recolección selectiva, aprovechamiento y cumplimiento de normatividad. Se aplicaron encuestas a establecimientos de 14 municipios de la provincia cuyas actividades comerciales estuvieran relacionadas con la distribución, el almacenamiento o el montaje de llantas. Se identificó desconocimiento del tema por parte de los administradores 
de los establecimientos evaluados y falta de acercamiento por parte de los proveedores de estos municipios. Aunque existen estrategias que permiten realizar un manejo adecuado de las llantas usadas, se hace necesaria la inclusión de los diferentes actores involucrados, así como el aumento de incentivos o beneficios para empresas que realicen este proceso de manera apropiada, o para quienes realicen actividades de reutilización, lo cual favorecería la gestión adecuada de este residuo.

Palabras clave: aprovechamiento, disposición, gestión de residuos, neumático de desecho, neumático fuera de uso.

\section{ABSTRACT}

In Colombia, used tires are not considered hazardous waste despite being composed of substances with this connotation, which generate negative impacts on health and the environment in case proper management is not performed. Although national regulatory instruments for selective collection systems have been generated, there is little knowledge of aspects related to their management, generation and handling; a situation that increases in low complexity municipalities where - despite its generation - adequate monitoring is not carried out and the supply of collection companies or collection centers is limited. Hence, a study on the management of used tires in low complexity municipalities of the Central Province of the department of Boyacá was carried out in order to inquire about their generation, disposal and management, as well as on the knowledge of selective collection programs, their use, and regulations compliance. Surveys were applied to establishments in 14 municipalities of the province whose commercial activities were related to tires distribution, storage or assembly. Lack of knowledge on the studied subject was identified in the administrators of the evaluated establishments, as well as a lack of rapprochement by tire suppliers of these municipalities. Although there are strategies that allow proper management of used tires, it is necessary to include the different actors involved and increase the incentives or benefits for companies that carry out this process properly, or for those who participate in reuse activities, which would favor the proper management of this type of waste.

Keywords: Recovery, disposal, waste management, waste tire, end of life tire.

\section{INTRODUCCIÓN}

Son consideradas Ilantas usadas aquellas de todos los diámetros que se encuentran en el mercado y que han dejado de ser útiles por haber perdido o disminuido las funciones principales para las cuales fueron diseñadas. De igual forma, se categorizan como estos residuos las llantas incompletas o pedazos de ellas que ya no son de utilidad, o que fueron reemplazadas por considerarse inseguras o de desecho (SEMARNAT, 2012). En la literatura referente al manejo o el aprovechamiento de este residuo, el término "Ilanta" se utiliza indistintamente de neumático. Para el presente estudio, se utilizará el primero, de acuerdo a lo establecido en la normatividad colombiana, la cual define llanta usada como la que técnicamente ha finalizado su vida útil y es susceptible de reencauche o aprovechamiento (MADS, 2017).

Aunque en Colombia las Ilantas no son consideradas un residuo peligroso (MADS, 2021), estos productos están compuestos de un gran número de sustancias con connotaciones peligrosas, las cuales generan impactos negativos sobre la salud y el ambiente si no se realiza un manejo apropiado. Algunos de los principales impactos asociados a la generación de este residuo son la quema a cielo abierto, práctica que genera contaminantes atmosféricos como material particulado, monóxido de carbono, óxidos de nitrógeno y compuestos orgánicos volátiles, entre otros (Casolco et al., 2013). 
Tales efectos se producen también en sistemas artesanales donde estos residuos se emplean como combustible alterno, debido a que se trata de emisiones no controladas en ambientes con escasa ventilación y exposición continua, como el caso de las hornillas paneleras en la región Andina colombiana, donde la llanta resulta ser un energético atractivo debido a su bajo costo y alto poder calorífico; a pesar de que la exposición de las personas a este tipo de emisiones genera efectos crónicos en su salud (CCB \& DAMA, 2006).

Adicionalmente, se conocen problemas como la generación de un aspecto visual negativo cuando son dispuestas de manera inadecuada, ya que por su volumen requieren amplios espacios de acopio (Casolco et al., 2013), o que por su alto poder calorífico podrían generar un incendio difícil de controlar (ANDELLAC et al., 2013). Debido a su estructura, estos residuos almacenan agua en su interior, lo que favorece la propagación de vectores y plagas nocivas que transmiten enfermedades como la fiebre amarilla o la encefalitis equina, que han generado un grave problema en muchos países, en especial por la propagación y eficiente dispersión de Aedes albopictus, importante vector transmisor del dengue (Casolco et al., 2013; RúaUribe et al., 2012).

Es así como se evidencia la necesidad de implementar acciones tendientes a proteger el medio ambiente y la salud humana mediante la prevención de la generación de estos residuos o la reducción de los posibles impactos adversos que pueda causar el manejo inadecuado de llantas usadas, por lo cual en Colombia se han propuesto algunos requisitos legales y normativos que promueven la ejecución de actividades dirigidas a minimizar la problemática causada por estos residuos, como la Resolución 1457 de 2010 (MAVDT, 2010), por la cual se establecieron los sistemas de recolección selectiva y gestión ambiental de llantas usadas (SRS) para favorecer su reciclaje, aprovechamiento como agregado asfáltico y reencauche, o para evitar que sean quemadas en espacios a cielo abierto o usadas como combustible en actividades informales.

En consecuencia, el MADS avanzó en el fortalecimiento de dichos sistemas, considerando, entre otros aspectos, el aumento en la generación de este residuo debido al crecimiento del parque automotor y la necesidad de involucrar otros tipos de llantas, como las de motocicletas, bicicletas y llantas fuera de carretera, producto de lo cual se generó la Resolución 1326 de 2017 (MADS, 2017), que establece la obligación de formular, presentar, implementar y mantener actualizados los SRS, a cargo de los productores de las llantas que se comercializan en el país, con el fin de prevenir y controlar la degradación del ambiente. Sin embargo, como mencionan Vélez y Echeverri (2016), la normatividad se ha quedado corta a la hora de establecer responsables y responsabilidades.

Por otra parte, la información en cuanto al manejo de llantas usadas a nivel regional es insuficiente. Los reportes de los últimos años han sido generados por la Corporación Autónoma Regional (Corpoboyacá), como autoridad ambiental en jurisdicción de la Provincia Centro, relacionando solo dos empresas debidamente registradas como gestores de llantas usadas, las cuales se encuentran ubicadas en el municipio de Duitama (Boyacá) (Corpoboyacá, 2018). Así mismo, el informe de gestión vigencia 2016 relaciona que en la ejecución del programa integral de residuos peligrosos se recolectaron 2.444 unidades de llantas desechadas a través de campañas de recolección (Corpoboyacá, 2016). Al respecto, la Superintendencia de Servicios Públicos Domiciliarios (SSPD, 2017) relaciona que Boyacá solo cuenta con dos estaciones de clasificación y aprovechamiento, ubicadas en los municipios de Tunja y Duitama, con una capacidad de almacenamiento de 154 y 40 toneladas, respectivamente, lo cual refleja una limitación en la oferta de servicios de recolección y almacenamiento temporal de Ilantas usadas, así como posibilidades de aprovechamiento.

En este contexto, se realizó un estudio sobre la gestión de llantas usadas en municipios de baja complejidad de la Provincia Centro del departamento de Boyacá (Colombia) con el fin de indagar acerca de su generación, disposición y manejo, al igual que sobre el conocimiento de programas de recolección selectiva, aprovechamiento y cumplimiento de normatividad. 


\section{MATERIALES Y MÉTODOS}

\section{Área de estudio}

El área de estudio corresponde a 14 municipios de baja complejidad de la Provincia Centro del departamento de Boyacá (Colombia), que a segundo semestre de 2016 registraban el transporte como una de las tres principales actividades económicas representativas en el Esquema de Ordenamiento Territorial: Cómbita, Cucaita, Chivatá, Chíquiza, Motavita, Oicatá, Samacá, Siachoque, Sora, Soracá, Sotaquirá, Toca, Ventaquemada y Tuta. Para el mismo período, estos municipios se encontraban categorizados por la Contaduría General de la Nación en nivel 6, de acuerdo con la Ley 1551 (Ley 1551 del Congreso de la República de Colombia, 2012), por presentar población menor a 10.000 habitantes e importancia económica grado 7 , con ingresos corrientes de libre destinación anuales no superiores a 15.000 salarios mínimos legales mensuales, por lo cual son considerados de baja complejidad.

\section{Recolección de datos}

Teniendo en cuenta el enfoque de investigación descriptiva (Tamayo, 2004), se realizó la recolección de información primaria a través de la visita de 52 establecimientos ubicados en los municipios mencionados, los cuales fueron seleccionados teniendo en cuenta 2 criterios: actividad vigente para el año 2016 y actividad comercial relacionada con la distribución, almacenamiento o montaje de llantas en sus diferentes categorías. El municipio de Tunja, capital del departamento, que también forma parte de la Provincia Centro, no fue incluido por ser de alta complejidad, y sus resultados se presentan en el trabajo de Bernal-Figueroa et al. (2021). En cada establecimiento se aplicó una encuesta estructurada con aspectos asociados a la gestión de llantas usadas. Además, se realizó la consulta de fuentes secundarias de información relacionadas con la gestión de llantas usadas en el ámbito nacional e internacional.

\section{RESULTADOS Y DISCUSIÓN}

Con referencia a las actividades que realizan los establecimientos que generan llantas usadas en la
Provincia Centro del departamento de Boyacá, se encontró que $29 \%$ de estos se dedican a actividades varias como el cambio, venta y reencauche de llantas, mientras que el porcentaje restante ejecuta algunas de estas actividades de forma separada (figura 1A). De igual forma, se identificó que los establecimientos no realizan un manejo adecuado a este residuo, considerando que los encuestados mencionan no haber recibido información o capacitaciones por parte de las autoridades ambientales, productores o comercializadores de llantas que les permitan realizar un manejo efectivo del residuo. Al respecto, la Autoridad Nacional de Licencias Ambientales de Colombia (ANLA, 2021) establece que los SRS formulados por los productores deberán especificar la cobertura inicial y las proyecciones se tendrán hasta lograr cubrir el 100 $\%$ de los municipios en donde se hayan comercializado los productos de los participantes del sistema. De igual forma, como refieren Taboada et al. (2013), las estrategias de las autoridades para el manejo de residuos deben ser acordes con las necesidades, la cultura y los recursos de las comunidades, así como con las características y volúmenes de los residuos generados.

De acuerdo con la información suministrada en cuanto a la generación de llantas usadas, se tiene que mensualmente el $40 \%$ de los establecimientos evaluados indica generar menos de 15 unidades, 39 $\%$ entre 16 y 50 y $21 \%$ más de 50 unidades (figura 1B), lo que da cuenta de una generación considerable de este residuo. Sin embargo, no se cuenta con datos públicos que permitan identificar la cantidad y el tipo de llantas usadas que se generan tanto en estos municipios como a nivel departamental. El informe de gestión 2016 de Corpoboyacá relaciona que se colectaron 2.444 unidades a través de campañas realizadas en la capital del departamento (Corpoboyacá, 2016), mientras que no se ha reportado su ejecución en los municipios considerados de baja complejidad, donde también se generan cantidades significativas. De igual forma, no se cuenta con información o indicadores exactos para establecer la cantidad y el tipo de llantas usadas dispuestas en sitios inadecuados (Fonseca \& Mesa, 2017), lo que limita la identificación de posibilidades de aprovechamiento para las Ilantas generadas en estos municipios. 
Figura 1. Resultados obtenidos de la aplicación de encuestas

\section{A. Actividades que realizan los establecimientos}

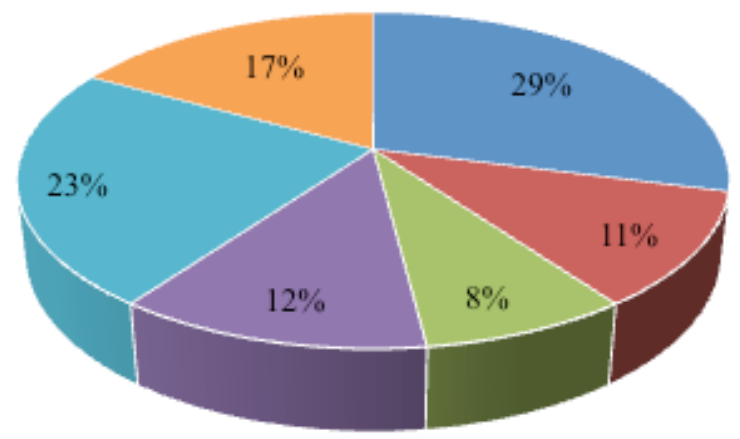

$=$ Todas

" Cambio y reencauche de llantas

- Cambio y venta de llantas
- Cambio de llantas

- Venta de llantas

" Reencauche de llantas

\section{Tipo de llantas que generan los establecimientos}

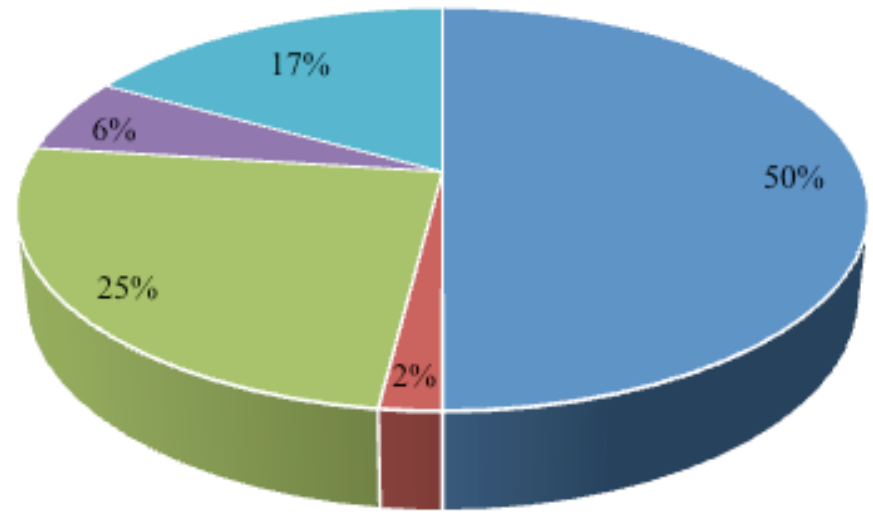

" Rin 13,14 "Camión "Moto - Bicicleta " Bus - Buseta 
B. Promedio de llantas usadas generadas mensualmente en los establecimientos

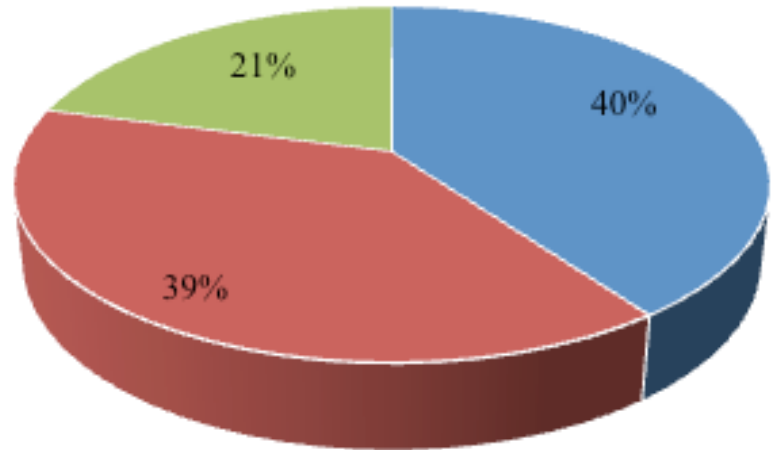

"Menos de 15 "Entre 16 y 50 "Mas de 50

\section{Disposición de llantas usadas}

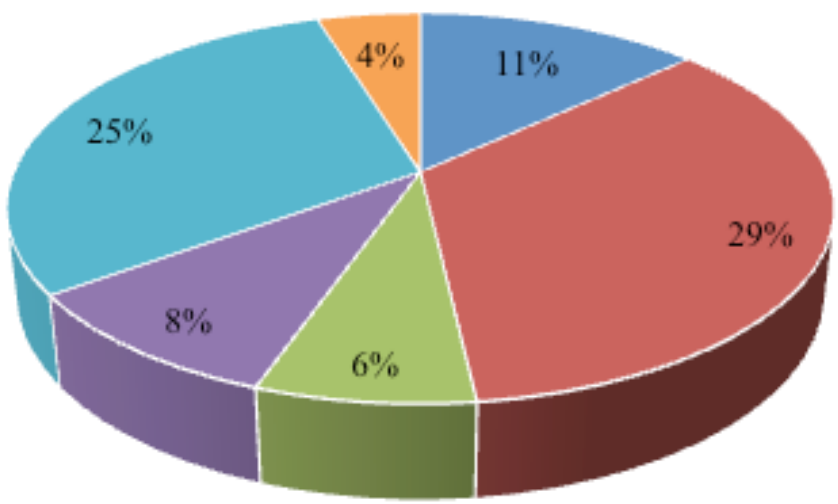

" Cliente

" Entrega a empresa de servicios públicos

" Almacena

" Entrega a entidades para reuso

" Reusa 
Frente al tipo de llanta generada, el $50 \%$ corresponde a llantas pequeñas de rines 13 y 14 (figura $1 \mathrm{C}$ ), lo cual, de acuerdo con Espinoza et al. (2020), está relacionado con que estas son las utilizadas para automóviles; por ende, se evidencia su mayor generación. La problemática relacionada con la alta generación de este residuo no solo se evidencia en estos municipios, sino que este fenómeno registra un volumen creciente a nivel mundial (Acosta et al., 2013; Casolco et al., 2013; Mmereki et al., 2019; Oboiriena \& North 2017; Song et al., 2018). Oboiriena y North (2017) mencionan que alrededor de 2.700 millones de unidades de neumáticos se produjeron en 2017, mientras que Acosta et al. (2013) indican que en Colombia la generación de residuos de llantas de automóvil, camioneta, camión y buseta se estimó en 61.000 t/año. Así mismo, Aragón y Espitia (2017) señalan que en Bogotá cerca de 2,5 millones de llantas cumplen su vida útil por año, por lo cual manejar adecuadamente estos desechos se ha convertido en un desafío (Song et al., 2018), del que la zona centro del departamento de Boyacá no es ajena.

Acerca del almacenamiento, el $57 \%$ de los establecimientos evaluados manifestó que no cuenta con un lugar específico para el acopio, mientras que el porcentaje restante indica que las almacena en lugares sin techos o sin paredes, lo que contribuye al estancamiento de aguas lluvias en su interior, así como a la alteración visual del paisaje, como se observa comúnmente. Córdova y Romo (2012) mencionan que en América Latina es habitual encontrar llantas usadas en la vía pública, en talleres mecánicos de automóviles, casas, cauces de arroyos y ríos, o la mayoría dispuestas en sitios ilegales. Además, en muchos casos se evidencia la quema abierta para la extracción del acero, creando un grave problema de contaminación ambiental (Acosta et al., 2013; Marín, 2012).

De igual forma, Aragón y Espitia (2017) manifiestan que en la ciudad de Bogotá las llantas usadas terminan en calles, humedales, e incluso en espacios residenciales, representando un problema estético, sanitario y medioambiental. Por su parte, Mondelo et al. (2014) indican que el apilamiento es el método usado históricamente en numerosos países, tanto industrializados como en vía de desarrollo, lo cual fue evidenciado en los sitios visitados. Respecto al tiempo de permanencia en estos sitios de almacenamiento, el $80 \%$ de los entrevistados manifiesta que los residuos de este tipo permanecen aproximadamente de 1 a 2 meses allí, para posteriormente ser llevados a otros lugares para su disposición final, lo cual se configura en una práctica inadecuada, considerando las problemáticas ambientales relacionadas. Estos establecimientos no cuentan con la estructura física adecuada para el almacenamiento temporal ni con puntos de recolección permanentes o cercanos, motivo por el cual recurren a dicha práctica. Otro aspecto que es importante mencionar frente a su inadecuado almacenamiento es la proliferación de vectores de enfermedades. Frente a ello, Mondelo et al. (2014) manifiestan que la falta de control hace que estas llantas se conviertan en criaderos potenciales de vectores debido a que pueden convertirse en recipientes contenedores de agua y, por consiguiente, en criaderos de mosquitos.

Con respecto a su disposición final, el $29 \%$ de los establecimientos indica que realiza su entrega a las empresas de servicios públicos y el 25 \% las reúsa, mientras que el $11 \%$ comenta que los clientes las conserva (figura 1D). Sobre el tema, en Ciudad de Juárez (México), Córdova y Romo (2012) encontraron que casi el $40 \%$ de las llantas de desecho se deposita en vulcanizadoras, cerca de la mitad en agencias y el porcentaje restante se almacena en casa como basura doméstica, o bien se arrojan a la vía pública, lo que representa un problema técnico, económico, ambiental y de salud pública (Córdova \& Romo, 2012; Casolco et al., 2013), por lo que las autoridades regionales han realizado campañas para su recolección. No obstante, como señalan Córdova y Romo (2012), esto no ha sido suficiente para resolver el problema, evidenciando la necesidad de buscar alternativas que incluyan al sector empresarial, la sociedad civil y la academia como parte de una sociedad consciente de buscar soluciones de manera coordinada.

En este sentido, el informe de Gestión de la Alcaldía Mayor de Tunja (2019) menciona que durante el período 2016-2019 se realizaron 6 jornadas de recolección de residuos especiales que permitieron colectar 4.726 unidades de llantas en esta ciudad. Sin embargo, no se hallaron datos públicos de procesos de recolección o cantidades para los municipios estudiados, lo cual evidencia la necesidad de realizar diagnósticos específicos en cuanto a la generación, a fin de identificar posibilidades de manejo y aprovechamiento para las zonas evaluadas. 
Por otra parte, se encontró que el $68 \%$ de los establecimientos conocen alternativas de aprovechamiento de llantas usadas como el reencauche, la trituración mecánica y el uso asfáltico. Sin embargo, el 32 \% restante desconoce las alternativas existentes. Al respecto, Chi y Tuyub (2015) mencionan que hace años la solución era botar estas llantas a la basura, las cuales eran llevadas a rellenos sanitarios para ser enterradas. No obstante, cuando se empezó a sensibilizar sobre temas ambientales, se encontró que estas son un desecho no biodegradable de larga vida. Córdova y Romo (2012) indican que para muchos usuarios lo más sencillo es conservar estos residuos en el patio trasero de sus hogares, mientras que otros los disponen en la vía pública y algunos más pagan por su disposición final (en México o Estados Unidos). A pesar de ello, estos autores resaltan que ninguna de estas opciones es una solución.

Otro de los grandes problemas identificados en la zona evaluada es el desconocimiento de puntos de recolección de este residuo, teniendo en cuenta que cerca del $94 \%$ de los evaluados manifiesta no conocerlos. Este aspecto ya ha sido contemplado en países europeos, donde, de acuerdo con Díaz et al. (2004), se generan incentivos a partir de una gran cantidad de puntos de recolección en las ciudades, que son posibles por una regulación exigente en términos de recolección y demanda de la sociedad por la implementación de medidas que contribuyan a proteger el ambiente y a depender menos de los vertederos para gestionar sus residuos. Garzón y Hernández (2014) señalan que el establecimiento de puntos de recolección se hace atractivo para gestores públicos o privados, pero también permitiría a los ciudadanos conocer la red y usarla de manera intensiva e intuitiva, generando conciencia social sobre su existencia y sobre los asuntos ambientales.

De otro lado, al indagar si los entrevistados consideran que el reciclaje de llantas contribuye al cuidado del medio ambiente, el $98 \%$ sostuvo que sí. De estos, 50 \% cree que ayuda a la eliminación de contaminantes emitidos a la atmósfera al evitar su quema, $17 \%$ porque se eliminan vectores e insectos y un 11 $\%$ porque mejora el paisaje y disminuye el riesgo de incendios (figura 2). Frente a la percepción acerca del aprovechamiento de las llantas usadas, el 77 \% señala que es una buena alternativa para disminuir el impacto ambiental y un $19 \%$ lo considera como una oportunidad de negocio. Sin embargo, también manifiestan desconocer el tema, y resaltan que es importante brindar información a los propietarios de los establecimientos y consumidores sobre las diferentes alternativas existentes.

Figura 2. Percepción de los establecimientos frente a la contribución del reciclaje de llantas usadas al cuidado del medio ambiente

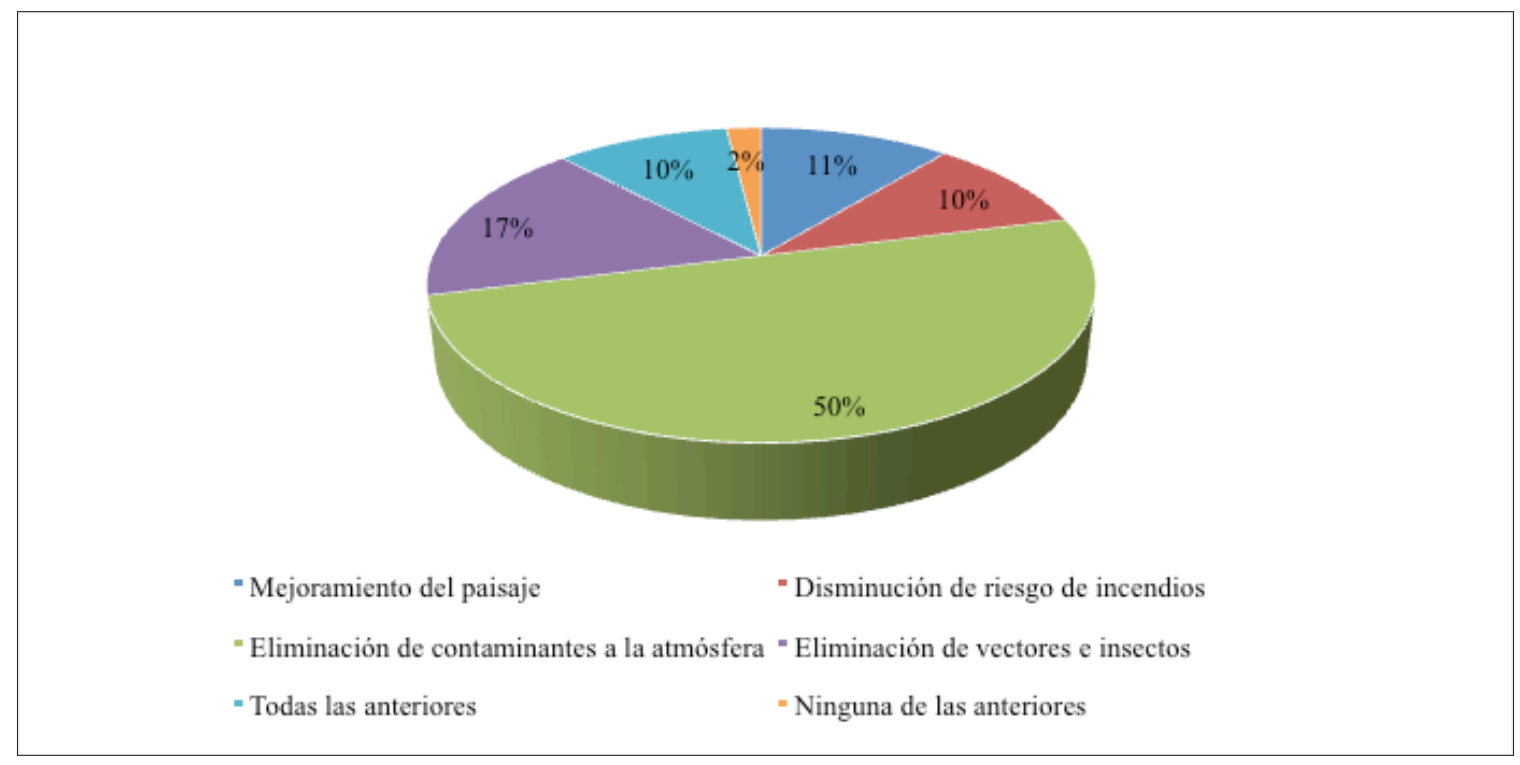


Actualmente existen múltiples investigaciones acerca de las alternativas de aprovechamiento de llantas usadas una vez han sido colectadas (Blanco et al., 2017; Mmereki et al., 2019; Oboiriena \& North 2017; Ospina \& Villada, 2011; Peláez et al., 2017; Song et al., 2018). En estudios nacionales, Peláez et al. (2017) presentan una revisión de las aplicaciones de caucho reciclado, centrándose en alternativas viables para medianas y pequeñas empresas, concluyendo que uno de los principales campos potenciales es utilizar este material como modificador de pavimentos y concretos, así como la posibilidad de usarlo en sistemas de adsorción de contaminantes en medios acuosos, techos ecológicos y la fabricación de celdas de combustible para la generación de energía. Ospina y Villada (2011) revisan los métodos para caracterizar combustibles líquidos y gaseosos obtenidos de llantas en desuso, señalando que en Europa, Asia y Norteamérica se cuenta con desarrollos tecnológicos para la optimización y el aprovechamiento de este residuo, mientras que en Colombia es aprovechable principalmente para la fabricación de suelas de zapatos, canchas sintéticas y pavimentación. Por su parte, Marín (2012) indica que las llantas usadas pueden ser empleadas para la producción de carbón activado de buena calidad, utilizado para la remoción de contaminantes en el agua, mientras que Miranda et al. (2006) exponen que estos residuos son una buena alternativa para la generaciónde energía debido a su alto poder calorífico.

Miranda et al. (2006) encontraron que el uso de una membrana absorbente en los neumáticos desechables resultó ser un método eficaz de drenaje de agua, contribuyendo a evitar posibles criaderos de $A$. aegypti. Además, Acosta et al. (2013) indican que la pirólisis se ha planteado como uno de los procesos tecnológicos apropiados para su aprovechamiento y valorización. Por otra parte, Mondelo et al. (2014) manifiestan que este tipo de residuos pueden utilizarse para regeneración de caucho, generación de energía o fabricación de nuevos materiales.

En el contexto internacional se identifica un importante número de estudios relacionados con el reciclaje y aprovechamiento de este residuo. Mohajerani et al. (2020) realizaron en Australia una investigación tendiente a utilizar la goma de llantas usadas como un componente del hormigón para reemplazar el uso de arena, mejorando con ello la ductilidad y resistencia del hormigón y haciendo posible su uso en la ingeniería geotécnica para el control de movimientos de tierra. Bowles et al. (2020) indican que dependiendo del tamaño de la goma se puede adicionar $5 \%$ del caucho reciclado para la elaboración de una llanta nueva. Kaliyappan et al. (2020) destacan que es posible desarrollar un compuesto híbrido de caucho natural-neopreno utilizando diferentes rellenos de residuos de caucho y carbón, incluyendo un efecto de variación de negro de humo sobre la morfología y las propiedades mecánicas del compuesto fabricado.

Arabiourrutia et al. (2020), en España, buscaron determinar cómo se puede hacer la valorización de neumáticos mediante pirólisis catalítica, demostrando que mediante esta técnica se pueden obtener subproductos de la llanta como gases, aceite y carbón. Mavukwana et al. (2020) estudiaron en Sur África la forma en que estos residuos se pueden convertir en electricidad basados en análisis de termodinámica. Ma et al. (2020) proponen en China el uso de partículas de goma de llantas usadas para la preparación de compuestos de cemento de fosfato y magnesio, los cuales son usados en ingeniería de carreteras y puentes. Además, Thai et al. (2020) estudiaron la conversión de fibras de caucho de residuos de neumáticos en aerogeles. Lo anterior por destacar algunos de los múltiples estudios que se han realizado para fomentar el aprovechamiento de este residuo en el contexto nacional y mundial, avances que podrían ser investigados para su aplicación en Colombia. No obstante, de acuerdo con Sebola et al. (2018), aunque las tecnologías e innovaciones para recuperar llantas al final de su vida útil están bien establecidas, el reciclaje de llantas de desecho no tiene éxito a nivel mundial.

Por último, es importante resaltar que el $92 \%$ de los establecimientos encuestados manifiestan no haber recibido alguna capacitación u obtenido información por parte de los proveedores, por lo cual es importante que se realicen acercamientos con estos pequeños municipios, de tal forma que los propietarios y trabajadores de los establecimientos puedan conocer cómo se debe realizar adecuadamente su disposición final, una vez las llantas hayan perdido su utilidad. De igual forma, el $54 \%$ de los establecimientos evaluados manifestó no contar con un plan de contingencia, por lo que resulta importante motivarlos a contar con planes que incluyan medidas de prevención y atención de emergencias que puedan generarse por el inadecuado almacenamiento de este residuo. 


\section{CONCLUSIONES Y RECOMENDACIONES}

En este estudio se identificó como mayor problema el desconocimiento de la gestión adecuada para el manejo de llantas usadas por parte de los administradores de los establecimientos evaluados, hecho que dificulta que las acciones contempladas por los entes gubernamentales sean implementadas eficientemente. Así mismo, se evidenció la falta de acercamiento de los proveedores de llantas a los establecimientos de estos municipios dedicados a su comercialización.

Aunque existen estrategias que permiten realizar un manejo adecuado de las llantas usadas, se hace necesaria la inclusión de los diferentes actores involucrados, así como el aumento de incentivos o beneficios para las empresas que realicen este proceso de manera apropiada, o para quienes realicen actividades de reutilización, con lo cual se favorecería una gestión adecuada de este residuo tanto en el contexto regional cono nacional.

El aprovechamiento de llantas usadas es un campo por explorar, teniendo en cuenta que es posible recuperar los materiales que las componen y darles nuevos usos. Para ello, se requiere incrementar la investigación en el área, de tal forma que se promueva el desarrollo de proyectos ambientales sostenibles con estrategias de innovación, como ya ha sido desarrollado ampliamente en el contexto internacional.

Se evidencia además la necesidad de realizar estudios de caracterización de las llantas usadas que se generan en la Provincia Centro con el fin de analizar alternativas para su aprovechamiento y potenciales estrategias de manejo. Igualmente, se deben llevar a cabo estudios de viabilidad sobre costos ambientales asociados a la recolección, almacenamiento y entrega a gestores, o posibilidades de implementar desarrollos tecnológicos como su uso energético o la fabricación de nuevos materiales, entre otras alternativas ya disponibles en el país.

Finalmente, al indagar sobre aspectos relacionados con la generación, recolección, manejo, aprovechamiento y conocimiento de la normativa aplicable a este sector, se identifica la necesidad de trabajo articulado entre actores involucrados en la gestión de llantas usadas a nivel nacional y regional, puesto que aunque se cuenta con una normativa vigente para la recolección selectiva y la gestión de llantas usadas, esta no se articula con las necesidades identificadas en los pequeños municipios analizados ni incluye compromisos puntuales para prevenir y controlar la degradación del medioambiente. Lo anterior, considerando estrategias de trabajo conjunto entre productores o importadores, gestores, consumidores, autoridades municipales y distritales y autoridades ambientales, para que desde la fuente se fomente la reutilización sostenible de este tipo de residuos. De igual forma, es importante el trabajo conjunto a nivel de provincia encaminado a identificar estrategias técnicas, administrativas y políticas que permitan cumplir con lo dispuesto en la Resolución 1326 de 2017 en cuanto a los sistemas de recolección selectiva y la gestión ambiental de llantas usadas para esta región.

\section{REFERENCIAS}

Acosta, R., Moncada, S., Gauthier-Maradei, P., \& Nabarlatz, A. (2013). Estudio preliminar de la producción de aceite y carbón mediante pirólisis intermedia de caucho de llantas usadas. Revista de Investigaciones Universidad del Quindío, 24(1), 139-145.

Alcaldía Mayor de Tunja. (2019). Informe acta de gestión "Tunja en Equipo" 2016-2019. Alcaldía Mayor de Tunja. https://alcaldiatunja. micolombiadigital.gov.co/sites/alcaldiatunja/ content/files/000713/35645_2019_29dic_ informe_final_gestion_2016_2019_tunja_en_ equipo.pdf

Arabiourrutia, M., López, G., Artetxe, M., Álvarez, J., Bilbao, J., \& Olazar, M. (2020). Waste tyre valorization by catalytic pyrolysis - A review. Renewable and Sustainable Energy Reviews, 129, 109932. https://doi.org/10.1016/j. rser.2020.109932

Aragón, J., \& Espitia, J. (2017). Evaluación térmica de la sustitución del material de la capa de drenaje en techos verdes por llantas usadas - análisis experimental en el II periodo húmedo climático del 2016, Bogotá. Avances Investigación en Ingeniería, 14(1), 21-36.

Asociación Nacional de Distribuidores de Llantas y Plantas Renovadoras A. C. [ANDELLAC], Asociación Nacional de Importadores de Llantas 
A. C. [ANILLAC], \& Cámara Nacional de la Industria Hulera [CNIH]. (2013). Plan de Manejo de Neumáticos Usados de Desecho. Conforme a la NOM-161-SEMARNAT-2011. ANDELLAC, ANILLAC, \& CNIH. http://reciclallantas.org.mx/ wp-content/uploads/2016/02/Plan-de-Manejode-Neumaticos-Usados-de-Desecho-2015-.pdf

Autoridad Nacional de Licencias Ambientales de Colombia [ANLA]. (2021). Sistema de Recolección Selectiva y Gestión Ambiental de Residuos Llantas Usadas. ANLA. http://portal.anla.gov. co/sistema-recoleccion-selectiva-y-gestionambiental-residuos-srs-llantas-usadas

Bernal-Figueroa, A., Rocha-Gil, Z., Medina-Moreno, J., Casas-Martínez, Y., \& Buitrago-Ramírez, L. (2021). Gestión de llantas usadas en la ciudad de Tunja, Boyacá (Colombia). Rev. U.D.C.A Actualidad \& Divulgación Científica, 24(1), e1627. http://doi. org/10.31910/rudca.v24.n1.2021.1627

Blanco, E., Travieso, D., \& Andrade, J. (2017). Energetic valorization of waste tires. Renewable and Sustainable Energy Reviews, 68, 306-315. http://dx.doi.org/10.1016/j.rser.2016.09.110

Bowles, A., Fowler, G., O'Sullivan, C., \& Parker, K. (2020). Sustainable rubber recycling from waste tyres by waterjet: A novel mechanistic and practical analysis. Sustainable Materials and Technologies, 25, e00173. https://doi. org/10.1016/j.susmat.2020.e00173

Casolco, S., Guerra, A., Hortega, R., Castillo, S., \& Bolaños, J. (2013). Llantas: Situación actual y oportunidades locales para la ingeniería civil en Puebla, México. Vislumbra, Revista de Divulgación del Tecnológico de Monterrey, 1(2), 63-74.

Chi, M., \& Tuyub, J. (2015). Análisis de las propiedades físicas y eléctricas en compuestos a base de emulsiones asfálticas con elastómeros y partículas conductoras. Ingeniería, 19(3),157-167.

Congreso de la República de Colombia. (2012, julio 6). Ley 1551. Por la cual se dictan normas para modernizar la organización y el funcionamiento de los municipios. Diario Oficial N. ${ }^{\circ} 48483$.

Córdova, G., \& Romo, M. (2012). Gestión pública para solucionar un problema ambiental: manejo de llantas de desecho en Ciudad Juárez. Región y Sociedad, 24(53), 119-151.

Corporación Autónoma Regional de Boyacá [Corpoboyacá]. (2016). Informe de gestión Vigencia 2016. Corpoboyacá. https://www. corpoboyaca.gov.co/cms/wp-content/ uploads/2016/01/informe-de-gestion-2016-v2. pdf

Corporación Autónoma Regional de Boyacá [Corpoboyacá]. (2018). Listado de gestores de llantas usadas en jurisdicción de Corpoboyacá. Corpoboyacá. https://www. corpoboyaca.gov.co/tramites-y-servicios/ gestion-integral-de-residuos-especiales/ gestion-de-llantas-usadas/

Díaz, A., Álvarez, M., \& González, P. (2004). Logística inversa y medio ambiente. McGraw-Hill.

Espinoza, L., Fariño, J., Patricio, Y., \& Mayorga, M. (2020). Responsabilidad social y ambiental: Tratamiento y disposición final de llantas usadas en la Ciudad de Machala. Gestión en el Tercer Milenio, 23(45), 39-48. http://dx.doi. org/10.15381/gtm.v23i45.17405

Fonseca, M., \& Mesa, C. (2017). Modelo logístico de recolección de llantas para la ciudad de Tunja. Revista Vínculos, 14(2), 108-118. https://doi. org/10.14483/2322939X.13196

Garzón, M., \& Hernández, D. (2014). Logística inversa en ciudades, las redes de recolección de artículos en pos-consumo. Twelfth Latin American and Caribbean Conference for Engineering and Technology (LACCEI'2014). Excellence in Engineering To Enhance a Country's Productivity. Guayaquil, Ecuador.

Kaliyappan, P., Dhananchezian, M., \& Rajkumar K (2020). Utilization of waste tyre rubber and carbon black to develop a neoprene rubber hybrid composite. Materials today: Proceedings, 27(2), 724-728. https://doi.org/10.1016/j. matpr.2019.11.307

Ma, C., Zhao, B., He, Y., Li, F., Long, G., \& Du, Y. (2020). Preparation and properties of sustainable magnesium phosphate cement composites with recycled tire rubber particles. Journal of 
Cleaner Production, 262, 121253. https://doi. org/10.1016/j.jclepro.2020.121253

Marín, B. (2012). En favor del medio ambiente: de llantas vieja a carbón activado. Revista Universitas Científica, junio, 32-35.

Mavukwana, A., Fox, J., \& Sempuga, B. (2020). Waste tyre to electricity: thermodynamics analysis. Journal of Environmental Chemical Engineering, 8(4), 103831. https://doi.org/10.1016/j. jece.2020.103831

Ministerio de Ambiente y Desarrollo Sostenible de Colombia [MADS]. (2017). Resolución 1326 de 2017. Por la cual se establecen los sistemas de Recolección selectiva y Gestión Ambiental de Llantas usadas y se dictan otras disposiciones. MADS.

Ministerio de Ambiente y Desarrollo Sostenible de Colombia [MADS]. (2021). Llantas usadas. MADS. https://www.minambiente.gov.co/index. php/asuntos-ambientales-sectorial-y-urbana/ programas-posconsumo-existentes/llantasusadas\#: :text=Las\%20llantas\%20usadas\%20 no\%20son,y\%20como\%20combustible\%20en\%20 actividades

Ministerio de Ambiente, Vivienda y Desarrollo Territorial de Colombia [MAVDT]. (2010). Resolución 1457 de 2010. Por la cual se establecen los sistemas de Recolección selectiva y Gestión Ambiental de Llantas usadas y se dictan otras disposiciones. MAVDT.

Miranda, R., Segovia, C., \& Sosa, C. (2006). Pirólisis de llantas usadas: Estudio cinético. Ingenierías, 9(32), 8-16.

Mmereki, D., Machola, B., \& Mkokwe, K. (2019). Status of waste tires and management practice in Botswana. Journal of the Air \& Waste Management Association, 69(10), 1230-1246. https://doi.org/10.1080/10962247.2017.1279696
Mohajerani, A., Burnett, L., Smith, J., Markovski, S., Rodwell, G., Rahman, M., Kurmus, H., Mirzababaei, M., Arulrajah, A., Horpibulsuk, S., \& Maghool, F. (2020). Recycling waste rubber tyres in construction materials and associated environmental considerations: A review. Resources, Conservation and Recycling, 155, 104679. https://doi.org/10.1016/j. resconrec.2020.104679

Mondelo, R., Fabricio, E., Gauto, N., \& Hernández, N. (2014). Uso de membrana de drenaje para evitar acumulación de agua y posibles criaderos de Aedes aegypti (Linnaeus 1762) (Diptera: Culicidae) en neumáticos desechables. Revista Cubana de Medicina Tropical, 66(2), 210-218.

Oboiriena, B., \& North, B. (2017). A review of waste tyre gasification. Journal of Environmental Chemical Engineering, 5, 5169-5178. http:// dx.doi.org/10.1016/j.jece.2017.09.057

Ospina, J., \& Villada, S. (2011). Métodos para caracterizar combustibles líquidos y gaseosos obtenidos de llantas en desuso a través de las normas ASTM. Lámpsakos, 3(6), 23-31. https:// doi.org/10.21501/21454086.830

Peláez, G., Velásquez, S., \& Giraldo, D. (2017). Aplicaciones de caucho reciclado: una revisión de la literatura. Ciencia e ingeniería Neogranadina, 27(2), 27-50. http://dx.doi.org/10.18359/ rcin. 2143

Rúa-Uribe, G., Suárez-Acosta, C., \& Rojo, R. (2012). Implicaciones epidemiológicas de Aedes albopictus (Skuse) en Colombia. Revista Facultad Nacional de Salud Pública, 30(3), 328-337.

Sebola, M., Mativenga, P., \& Pretorius, J. (2018). A benchmark study of waste tyre recycling in South Africa to European Union Practice. Procedia CIRP, 69, 950-955. https://doi.org/10.1016/j. procir.2017.11.137 
Secretaría de Medio Ambiente y Recursos Naturales [SEMARNAT]. (2012). Guía para el establecimiento de un centro de acopio de llantas de desecho. SEMARNAT. https://www.sema. gob.mx/VENTANILLA/SGA/030\%20GUIA\%20 CENTRO\%20DE\%20ACOPIO\%20LLANTAS.pdf

Song, P., Zhao, X., Cheng, X., Li, S., \& Wang, S. (2018). Recycling the nanostructured carbon from waste tires. Composites Communications, 7, 12-15. https://doi.org/10.1016/j.coco.2017.12.001

Superintendencia de Servicios Públicos Domiciliarios de Colombia [SSPD]. (2017). Informe Nacional de Aprovechamiento 2016. SSPD. https://www. superservicios.gov.co/sites/default/archivos/ Noticia/Noticias\%20de\%20interes/2018/ Sep/187302.pdf

Taboada, P., González, Q., Aguilar, V., Cruz, S., \& Ramírez, S. (2013). Manejo y potencial de recuperación de residuos sólidos en una comunidad rural de México. Revista Internacional de Contaminación Ambiental, 29(3), 43-48.
Tamayo, M. (2004). El proceso de la investigación científica (4⿳亠口了 ed.). Limusa.

Thai, Q., Chong, R., Nguyen, P., Le, D., Le, P., PhanThien, N., \& Duong, H. (2020). Recycling of waste tire fibers into advanced aerogels for thermal insulation and sound absorption applications. Journal of Environmental Chemical Engineering, 8(5), 104279. https://doi.org/10.1016/j. jece.2020.104279

Vélez, D., \& Echeverri, J. (2016). Caracterización de la generación de neumáticos y manejo de sus desechos en el departamento del Quindío. Revista Aletheia, 6, 31-47. 